\title{
On the problem of emergence of classical space-time: The quantum-mechanical approach
}

\author{
Alexey A. Kryukov \\ University of Wisconsin, UWC Department of Mathematics, 780 Regent Street, \\ Madison, WI 53708 \\ E-mail: akriouko@uwc.edu
}

\begin{abstract}
The classical space-time structure is derived from the structure of an abstract infinite dimensional separable Hilbert space $\mathbf{S}$. For this $\mathbf{S}$ is first realized as a Hilbert space $H^{*}$ of functions of abstract parameters. Such a realization is associated with the process of measuring position of macroscopic particles naturally occurring in the universe. The process of decoherence and collapse induced by the measurement is in return associated with the choice of a "decohered" submanifold $M$ of realization $H^{*}$. The submanifold $M$ is then identified with the classical space-time. The mathematical formalism is developed which permits to recover the usual Riemannian geometry on space-time in terms of the Hilbert structure on $\mathbf{S}$. The specific functional realizations of $\mathbf{S}$ are shown to produce space-times of different geometry and topology.
\end{abstract}

PACS numbers: 03.65Ta, 03.65Yz, 02.40-k 


\section{Introduction}

We take for granted that physical events take place in space-time. Mathematically this is reflected in realization of physical quantities as functions of space-time points. The shortcomings of this are well known. In particular, position of a particle in quantum field theory (QFT) is only defined to energies less than the particle's mass. The field description of the smaller distances leads to inconsistencies which up until now have not been completely resolved.

By now the string/M-theory is generally believed to be the leading successor of the QFT. Not only it deals successfully with divergencies plugging QFT, but it also leads to a unified approach to the known interactions. However, one of the main objections to the string/M-theory is that it requires the notion of space-time to begin with. In particular, the theory, while deducing gravity, does not deduce the space-time which therefore stays classical.

The situation is reminiscent of the quantum mechanics (QM). The latter theory also seems to require the classical behavior of the measuring devices for its mere existence. The fundamental problem to deduce the classical world from the quantum one is therefore common to both theories.

Motivated by analysis of position measurement experiments in QM we introduce here a model in which the classical space-time is considered to be a subspace of an abstract infinite-dimensional Hilbert space $\mathbf{S}$. The space $\mathbf{S}$ is associated with the space of states of a macroscopic test-particle in the universe. A specific realization of $\mathbf{S}$ as a space of functions is then associated with a particular measurement performed on the particle. A natural measurement performed on macroscopic particles in the universe is the measurement of their positions. Respectively, the space $\mathbf{S}$ is naturally realized by a Hilbert space $H^{*}$ of linear combinations of localized state functions. These functions are considered in the paper as functions of abstract parameters, so that no pre-existing notion of space-time is required. The process of decoherence and collapse induced by the measurement is associated with a choice of submanifold $M$ of $H^{*}$ consisting of localized normalized state functions. The resulting space $M$ can be then identified with the classical space-time.

In following this line of thought we also develop a formalism which permits to reduce in a natural way the infinite-dimensional differential geometry on $\mathbf{S}$ to the ordinary Riemannian geometry on space-time. This reduction can be also associated with the large-scale measurement process naturally occurring in the universe. In this respect the space-time structure is shown to emerge as a result of position measuring experiments constantly performed in/on the universe. It is also shown that different topologies (let alone geometries) on space-time are readily available from the structure of a single

Hilbert space $\mathbf{S}$. Moreover, a particular choice of topology seems to be related to a particular measurement set up realized in the universe.

Despite these promising results, the paper cannot be considered as more than just a model of emergence. This is so because the entire construction is based on 
the non-relativistic quantum mechanical formalism and because additional simplifying assumptions have been made. The quantum field-theoretic approach to the problem together with a more refined treatment of the subject are left for a future publication.

\section{How to "pin" a point of space-time?}

The modern classical notion of space-time is due to Einstein. According to Einstein, the space-time is a four-dimensional Riemannian manifold. To accept such a manifold as physical one must be able to physically "pin" the points of space at any moment of time.

To pin a point is to observe an event at the point. Most typically the observations of this kind are done by means of scattering processes. To observe location of a macroscopic object, for example, one can observe the light scattered off the object. If one wants to be more precise in "fixing" a point, the scattering of high energy electrons off a test particle could be used instead.

In principle, position of a particle in the non-relativistic quantum mechanics can be measured as precisely as one wishes. Up to a constant factor the state function $\psi_{a}$ of the particle with a given position $a$ is the solution of the eigenvalue problem

$$
\widehat{x} \psi_{a}(x)=a \psi_{a}(x)
$$

for the operator of coordinate $\widehat{x}$. The non-normalized solutions of this equation are given by an arbitrary constant multiple of the delta-function. By fixing normalization we observe that position of a particle determines its state. On the other hand, if the state function of a particle is concentrated at a point in space, then position of the particle is known. That is, there exists a bijective correspondence between points in space and state functions of a particle localized at any one of these points. In this respect one can conclude that to "pin a point" is to identify the point with the state function of a particle localized at the point.

The resulting identification of the points in space with the state functions of a localized particle makes one wonder whether it would be fruitful to consider the classical space-time as a particular subspace of the space of states of the particle. This by itself could seem rather artificial and pointless without the following supporting circumstance: Macroscopic particles in the universe are found in well localized in space wave packets. Ideally, when the size of a packet is negligible, the state function of the particle is the delta-function. As macroscopic test-particles are the ones used to determine the largescale structure of space-time, it becomes quite plausible that the origin of the classical space-time can be traced back to the Hilbert space of quantum states.

In more detail, scattering processes on (non-interacting) macroscopic test-particles constantly happening in the universe can be identified with measuring position of the particles. This measurement leads to realization of the Hilbert space of states of each particle as a space $H^{*}$ of linear combinations of the localized (ideally, point supported) state functions. The process of decoherence triggered by the measurement destroys 
superpositions of the functions. That is, decoherence, together with the consecutive collapse (reduction of state) ensure spatial localization of the particles. In principle, assuming that we keep record of each position measurement, i.e. in case of the so-called selective description, the state of each particle stays pure. As a result, the points of space can be identified with the states of particles each particle being "attached" to a point.

To avoid dealing with tensor products of Hilbert spaces of states of individual particles we prefer here to identify the space-time with all possible states of a single localized test-particle. The single particle approach poses several questions which we do not address in the paper. A more careful analysis of the situation including the QFT treatment of the problem is left for a future publication. Here, motivated by the present analysis, we simply accept the embedding of the space-time into the Hilbert space $H^{*}$ as a hypothesis and explore its consequences.

Before moving on let us point out that mathematically the embedding of a finitedimensional manifold into an infinite-dimensional one is always possible. The question therefore is to find the embedding that would be physically meaningful and interesting. In particular, we want to use the embedding to derive the space-time structure from the Hilbert structure on $H^{*}$. Thus, the elements of $H^{*}$ shall not be assumed to be functions of space-time coordinates but shall be instead functions of abstract parameters. The already discussed measurement of position of a macroscopic particle shall be mathematically the process of reduction of $H^{*}$ to a submanifold consisting of "decohered" states. Such a submanifold shall be then identified with the classical space-time leading to the measurement induced emergence of space-time.

As the states of localized at a point particle are given in QM by the delta-function, it is important to comment on the nature of the space $H^{*}$. It is usually assumed that the delta-like states in QM cannot be elements of a Hilbert space of states. The existence of various Hilbert spaces of distributions demonstrates that this opinion is wrong. Moreover, in [5] we have developed a formalism that includes the improper and the square-integrable states on equal footing. For this the metric on Hilbert spaces of functions is made dependent on the variety of functions making up a particular space. The resulting formalism presents an alternative to the generally accepted rigged Hilbert space approach to improper states in QM (see [3]).

In particular, the space $H^{*}$ of real valued generalized functions "of" $x \in R^{4}$ finite in the metric

$$
(\varphi, \psi)_{H^{*}}=\int e^{-(x-y)^{2}} \varphi(x) \psi(y) d x d y
$$

can be shown to be Hilbert (see [5]). Such a space contains delta-functions as, for example,

$$
\int e^{-(x-y)^{2}} \delta(x) \delta(y) d x d y=1 .
$$

Moreover, $H^{*}$ contains all derivatives of delta-functions as well.

Throughout the paper Hilbert spaces with the metric defined by a smooth kernel $k(x, y)$ will be generically denoted by $H^{*}$. 
Let us now develop a mathematical formalism of emergence that satisfies the discussed criteria.

\section{The "Emergence formalism"}

In this section we make no initial assumption about existence of space-time. Our main object is an abstract separable Hilbert space $\mathbf{S}$. This space is taken to be a model of the "quantum" space which shall replace the classical space-time.

As discussed in the previous section, the natural large-scale measurement process in the universe is the process of measuring positions of macroscopic particles. As a result, the macroscopic particles in the universe are in eigenstates of the position operator $\widehat{x}$. As the space $\mathbf{S}$ is associated with the space of states of a macroscopic test-particle in the universe, it is naturally realized by a Hilbert space $H^{*}$ of linear combinations of the delta-functions.

Let us point out, however, that this quantum-mechanical picture is only used as a motivation. In particular, the (generalized) functions in $H^{*}$ are at this point functions of abstract parameters $a$ which are not assumed to be the space-time coordinates. In what follows we will assume that parameters $a$ take values in the abstract Euclidean space $R^{4}$.

A particular realization of $\mathbf{S}$ as a space $H$ of functions can be mathematically described by an isomorphism $e_{H}: H \longrightarrow \mathbf{S}$. Such an isomorphism will be called a functional basis on $\mathbf{S}$ (see [5]).

Assume in particular that, as before, $H^{*}$ is a Hilbert space generated by the deltafunctions. Then the isomorphism $e_{H^{*}}: H^{*} \longrightarrow \mathrm{S}$ will be called a natural functional basis on $\mathbf{S}$.

Let now $\Phi_{0}$ be a point of $\mathbf{S}$ and let $\Gamma_{0}$ be the algebra of differentiable functionals on a neighborhood of $\Phi_{0}$. That is, the functionals in $\Gamma_{0}$ are defined on the elements $\Phi$ which belong to a neighborhood of $\Phi_{0}$ in $\mathbf{S}$. Let $\Phi_{t}: R \longrightarrow \mathbf{S}$ be a differentiable path in $\mathbf{S}$ which passes through the point $\Phi_{0}$ at $t=0$.

The vector tangent to the path $\Phi_{t}$ at the point $\Phi_{0}$ is defined as a map $X: \Gamma_{0} \longrightarrow R$ given by

$$
X F=\left.\frac{d F\left(\Phi_{t}\right)}{d t}\right|_{t=0} .
$$

Such a definition of tangent vector is common in the finite dimensional setting. In case of the infinite number of dimensions a different approach is more common (see [1]). The definition (4) is, however, more adequate for our needs. The coordinate formalism of [5] makes it a convenient tool for developing differential geometry on infinite-dimensional manifolds.

As the functionals $F$ and the path $\Phi_{t}$ are assumed to be differentiable, we have:

$$
\left.\frac{d F\left(\Phi_{t}\right)}{d t}\right|_{t=0}=\left.\left.F^{\prime}(\Phi)\right|_{\Phi=\Phi_{0}} \Phi_{t}^{\prime}\right|_{t=0},
$$


where $\left.F^{\prime}(\Phi)\right|_{\Phi=\Phi_{0}}: \mathbf{S} \longrightarrow R$ is the derivative functional at $\Phi=\Phi_{0}$ and $\left.\Phi_{t}^{\prime}\right|_{t=0} \in \mathbf{S}$ is the derivative of $\Phi_{t}$ at $t=0$. As always, a tangent vector $X$ is a linear map satisfying the product rule.

Let us show that the space $\mathbf{T}_{\mathbf{0}} \mathbf{S}$ of various tangent vectors $X$ with an appropriate topology is isomorphic to $\mathbf{S}$. For this let $e_{H}$ be a functional basis on $\mathbf{S}$. For a path $\Phi_{t}$ through $\Phi_{0}$ let $\varphi=\varphi_{t}$ be an equation of the path in the $e_{H}$-basis (i.e. $\varphi_{t}=e_{H}^{-1}\left(\Phi_{t}\right)$ ). As $\left.\Phi_{t}^{\prime}\right|_{t=0} \in \mathbf{S}$, we also have $\varphi_{t_{0}}^{\prime} \in H$. Then

$$
X F=\left.\frac{d F\left(\Phi_{t}\right)}{d t}\right|_{t=0}=\left.\int \frac{\delta f(\varphi)}{\delta \varphi(x)}\right|_{\varphi=\varphi_{0}} \xi(x) d x,
$$

where $\xi=\left.\varphi_{t}^{\prime}\right|_{t=0}$ and the linear functional $\left.\frac{\delta f(\varphi)}{\delta \varphi(x)}\right|_{\varphi=\varphi_{0}}$, which is an element of the dual space $H^{*}$, can be thought of as the derivative functional $F^{\prime}$ in the basis $e_{H}$. The integral sign is understood here in the sense of action of $\frac{\delta f(\varphi)}{\delta \varphi(x)}$ on $\xi$ (see [5] for notations). In these notations we can also write

$$
X=\int \xi(x) \frac{\delta}{\delta \varphi(x)} d x
$$

where $\xi \in H$ and the right hand side acts on functionals $f$ defined by

$$
f(\varphi)=F(\Phi),
$$

where $F \in \Gamma_{0}$ and $e_{H} \varphi=\Phi$. In particular, we see that the coordinate formalism permits one to present tangent vectors in a mathematically rigorous way in the form similar to the finite dimensional case, i.e. as "linear combinations" of the "partial" derivatives.

Let us now consider the map $\omega: \mathbf{T}_{\mathbf{0}} \mathbf{S} \longrightarrow \mathbf{S}$ which in the basis $e_{H}$ is given by $\left.\int \xi(x) \frac{\delta}{\delta \varphi(x)}\right|_{\varphi_{0}} d x \longrightarrow \int e_{H}(x) \xi(x) d x$. Here again, in accordance with notations used in [5], the integral on the right signifies the action of $e_{H}$ on $\xi$.

To show that $\omega$ is an isomorphism, consider an arbitrary element $\Psi=\int e_{H}(x) \eta(x) d x$ of $\mathbf{S}$ and a path $\varphi_{t}=\varphi_{0}+\eta t$. The vector tangent to this path at $t=0$ is $X=\int \eta(x) \frac{\delta}{\delta \varphi(x)} d x$. It follows that $\omega$ is surjective.

On another hand, if $X=\int \xi(x) \frac{\delta}{\delta \varphi(x)} d x=0$, then for any $F \in \Gamma_{0}$ we have $X F=\int \frac{\delta f(\varphi)}{\delta \varphi(x)} \xi(x) d x=0$. Since the derivative of a linear functional is the functional itself and since any continuous linear functional on $\mathbf{S}$ is an element of $\Gamma_{0}$ we conclude that $h(\xi)=0$ for any $h \in H^{*}$. It follows that $\xi=0$, that is, $\omega$ is injective.

The one-to-one linear map $\omega$ induces a Hilbert structure on the space $\mathbf{T}_{\mathbf{0}} \mathbf{S}$. Relative to this structure $\omega$ is an isomorphism of Hilbert spaces.

The space $\mathbf{T}_{\mathbf{0}} \mathbf{S}$ with the above Hilbert structure will be called the tangent space to $\mathrm{S}$ at the point $\Phi_{0}$.

For the tangent space $\mathbf{T}_{\mathbf{0}} \mathbf{S}$, the dual space $\mathbf{T}_{\mathbf{0}}^{*} \mathbf{S}$ is called the cotangent space at $\Phi_{0}$. The differential $d F$ of a functional $F \in \Gamma_{0}$ is an element of $\mathbf{T}_{\mathbf{0}}^{*} \mathbf{S}$ which satisfies

$$
d F(X)=X F
$$

for any $X \in \mathbf{T}_{\mathbf{0}} \mathbf{S}$. 
Notice that for a smooth enough $F$ the usual Taylor formula is valid. In particular, if $F$ is twice differentiable on a neighborhood of $\Phi_{0}$, then

$$
F\left(\Phi_{0}+\Xi\right)=F\left(\Phi_{0}\right)+d F\left(\Phi_{0}, \Xi\right)+\frac{1}{2 !} d^{2} F\left(\Phi_{0}, \Xi\right)+o(\|\Xi\|)\|\Xi\| .
$$

Here $d^{2} F\left(\Phi_{0}, \Xi\right)=F^{\prime \prime}\left(\Phi_{0}\right)(\Xi, \Xi), F^{\prime \prime}\left(\Phi_{0}\right)$ is the second derivative of $F$ which is a symmetric bilinear functional on $\mathbf{T}_{\mathbf{0}} \mathbf{S} \times \mathbf{T}_{\mathbf{0}} \mathbf{S}$, and $\|\Xi\|$ is the norm of $\Xi$ in $\mathbf{S}$.

Having discussed the general differential geometry on $\mathbf{S}$ let us return to the specific realization of $\mathbf{S}$ by a space $H^{*}$ of linear combinations of delta-functions. Recall that the corresponding functional basis is called natural. In accordance with the previous discussion we now identify the classical space-time $M$ with the subset of $H^{*}$ consisting of all delta-functions $\delta(x-a)$ for various possible $a \in R^{4}$.

Notice first of all that as a subset of $H^{*}, M$ is a topological space with the induced (subset) topology. We will further assume that the inclusion map $i: M \longrightarrow H^{*}$ is an embedding. In particular, $M$ will be a submanifold of $H^{*}$.

Clearly, $M$ is not a linear subspace of $H^{*}$. However, in the simplest case when $M$ is diffeomorphic to $R^{4}$, the space $M$ has an induced linear structure (different than the one on $\left.H^{*}\right)$.

As already discussed, the proposed choice of $M$ corresponds to a "decohered" universe. In this sense it is physically sound. However, it is also important to verify that the tangent bundle structure and the Riemannian structure on $M$ are naturally induced by the embedding of $M$ into $H^{*}$.

The notion of "naturality" requires some clarification. As already discussed, the embedding of the space-time into $H^{*}$ is always possible. Moreover, it is possible to ensure an isometric embedding, i.e. the Riemannian metric on $M$ will be just a restriction (pull-back) of the Hilbert metric on $H^{*}$. What we want in addition is a compatibility of differential-geometric "languages" on $M$ and $H^{*}$. More precisely, the variational derivatives used to define vectors tangent to $\mathbf{S}$ (or its realization $H^{*}$ ) according to (6), should naturally reduce to the partial derivatives that permit to define vectors tangent to $M$. Respectively, tensor algebra on spaces tangent to $\mathbf{S}$ (or $H^{*}$ ) must "project" to the tensor algebra on spaces tangent to $M$. In particular, the Hilbert metric on $\mathbf{S}$ (respectively, $H^{*}$ ) shall reduce to the Riemannian metric on $M$. In other words, we want to express the finite dimensional differential geometry on space-time in terms of the infinite-dimensional differential geometry on a Hilbert space.

For this, let us select from all paths in $H^{*}$ the "decohered" ones, i.e. the paths laying in $M$. For each value of the parameter $t$ any such path $f_{t}$ reduces to a delta-function. That is,

$$
f_{t}(x)=\delta(x-a(t))
$$

for some function $a(t)$ which takes values in $R^{4}$.

To find vectors tangent to such paths we proceed as before. Consider first the action of linear (continuous) functionals on $f_{t}$. Such functionals will be then elements 
of the space $H$ dual to $H^{*}$ and will be denoted by $\varphi$. We have:

$$
\varphi\left(f_{t}\right)=\int \varphi(x) \delta(x-a(t)) d x=\varphi(a(t)) .
$$

Respectively,

$$
\left.\frac{d \varphi\left(f_{t}\right)}{d t}\right|_{t=0}=\left.\left.\frac{\partial \varphi(x)}{\partial x^{\mu}}\right|_{x=a(0)} \frac{d a^{\mu}}{d t}\right|_{t=0}
$$

The expression on the right can be immediately identified with the ordinary 4-vector acting on the function $\varphi(x)$. In the more general non-linear case situation is similar. Consider for example a quadratic functional

$$
b\left(f_{t}\right)=\int b(x, y) \delta(x-a(t)) \delta(y-a(t)) d x d y=b(a(t), a(t)) .
$$

Then, as before

$$
\left.\frac{d b\left(f_{t}\right)}{d t}\right|_{t=0}=\left.\left.\frac{\partial b(x, x)}{\partial x^{\mu}}\right|_{x=a(0)} \frac{d a^{\mu}}{d t}\right|_{t=0} .
$$

Notice that the formula (13) and its nonlinear analogues can be obtained directly from (6). For example:

$$
\frac{d \varphi\left(f_{t}\right)}{d t}=\int \frac{\delta \varphi(f)}{\delta f(x)} \frac{d f_{t}}{d t} d x=-\int \varphi(x) \nabla_{\mu} \delta(x-a) \frac{d a^{\mu}}{d t} d x=\frac{\partial \varphi}{\partial x^{\mu}} \frac{d a^{\mu}}{d t}
$$

where linearity of the functional $\varphi$ along with the "integration by parts" formula have been used. Notice in particular the validity (in generalized sense) of the formula

$$
\frac{d f_{t}(x)}{d t}=-\nabla_{\mu} \delta(x-a) \frac{d a^{\mu}}{d t} .
$$

From (13) and its nonlinear analogues we conclude that elements of $\mathbf{T}_{\mathbf{0}} \mathbf{S}$ tangent to the curves (11) are "naturally" (in the above sense) equivalent to the ordinary 4-vectors.

Let us now see how the Riemannian metric on $M$ is induced by the embedding of $M$ into $H^{*}$.

Let us assume first that $H^{*}$ is a real Hilbert space. Let $k(x, y)$ be the kernel of the Hilbert metric $K: H^{*} \times H^{*} \longrightarrow R$. In particular, the norm of a vector $\delta f \in H^{*}$ can be written as

$$
\|\delta f\|_{H^{*}}^{2}=\int k(x, y) \delta f(x) \delta f(y) d x d y .
$$

Let us remark that we identify here the spaces tangent to $H^{*}$ with $H^{*}$ itself. Assuming now that $f=f_{t}=\delta(x-a(t))$ is a path in $M$ and therefore $\delta f(x)=\left.\frac{d f(x)}{d t}\right|_{t=0}$, and using (17) with $a=a(0)$ we have:

$\int k(x, y) \delta f(x) \delta f(y) d x d y=\left.\left.\int k(x, y) \nabla_{\mu} \delta(x-a) \frac{d a^{\mu}}{d t}\right|_{t=0} \nabla_{\nu} \delta(y-a) \frac{d a^{\nu}}{d t}\right|_{t=0} d x d y$.

"Integration by parts" in the last expression gives then

$$
\int k(x, y) \delta f(x) \delta f(y) d x d y=\left.\left.\left.\frac{\partial^{2} k(x, y)}{\partial x^{\mu} \partial y^{\nu}}\right|_{x=y=a} \frac{d a^{\mu}}{d t}\right|_{t=0} \frac{d a^{\nu}}{d t}\right|_{t=0} .
$$


By defining $\left.\frac{d a^{\mu}}{d t}\right|_{t=0}=d x^{\mu}$, we have

$$
\int k(x, y) \delta f(x) \delta f(y) d x d y=g_{\mu \nu}(a) d x^{\mu} d x^{\nu},
$$

where

$$
g_{\mu \nu}(a)=\left.\frac{\partial^{2} k(x, y)}{\partial x^{\mu} \partial y^{\nu}}\right|_{x=y=a} .
$$

As the functional $K$ is symmetric, its kernel $k(x, y)$ can be chosen to be a symmetric function of $x$ and $y$. Then, the tensor $g_{\mu \nu}(a)$ will be symmetric as well. If in addition

$\left.\frac{\partial^{2} k(x, y)}{\partial x^{\mu} \partial y^{\nu}}\right|_{x=y=a}$ is positive definite at every $a$, the tensor $g_{\mu \nu}(a)$ can be identified with a Riemannian metric on space-time. One could similarly obtain a metric of Lorentzian signature.

It is important to know whether an arbitrary Riemannian metric $g_{\mu \nu}(a)$ on spacetime can be obtained in such a way. Clearly, for a function $k(x)$ with $x \in R^{4}$ the form $\frac{\partial^{2} k(x)}{\partial x^{\mu} \partial x^{\nu}}$ is rather special and cannot be made equal to an arbitrary Riemannian metric on $R^{4}$. However, we have twice as many variables at our disposal.

To analyze the situation assume here that the space $H^{*}$ is a complex Hilbert space of (generalized) functions on $C^{4}$. The variables $x, y$ in (18) are then replaced with the complex conjugate variables $z, \bar{z}$. The Hilbert metric on $H^{*}$ is necessary Hermitian. This can be assured, in particular, by choosing a real-valued kernel $k(z)$. Moreover, the form $g_{\mu \bar{\nu}}=\frac{\partial^{2} k(z)}{\partial z^{\mu} \partial \bar{z}^{\nu}}$ is then automatically Hermitian as well. If in addition $g_{\mu \bar{\nu}}$ is positive definite, the Riemannian metric $g_{\mu \bar{\nu}}$ is known to be Kähler. Moreover, an arbitrary Kähler metric on $C^{4}$ can be written locally is such a way (see for example [2]).

In [6] it was verified that any real analytic Riemannian n-dimensional manifold can be locally isometrically embedded into a Kähler n-dimensional manifold. Together with the above, this result assures that any analytic Riemannian metric can be locally written in the form (22). Moreover, this also proves that the complex Hilbert space structure on $H^{*}$ naturally leads to a Kähler structure on the complex extension of space-time.

\section{Discussion and outlook}

Let us review the advocated scenario of emergence of the classical space-time.

We began with the observation that the large-scale structure of space-time can be naturally recovered from the Hilbert space of states of a macroscopic test-particle in the universe.

To use this fact in analyzing the process of emergence, we have dropped the assumption of a pre-existing space-time. Instead, the abstract infinite-dimensional separable Hilbert space $\mathbf{S}$ is taken to be a model of space-time adequate to the quantum theory.

To recover the classical space-time it is necessary to find, first of all, a specific realization of $\mathbf{S}$ by a space of functions. Such a realization can be associated with a particular measurement performed on a macroscopic particle in the universe. The typical such measurement is the continuous measurement of the particle's position. 
Respectively, the space $\mathbf{S}$ is naturally realized as a space $H^{*}$ generated by the deltafunctions $\delta(x-a)$, where $x, a \in R^{4}$. The coordinate formalism developed in [5] demonstrates that such a space can be made Hilbert by choosing a metric on $H^{*}$ to be a bilinear functional with a continuous kernel $k(x, y)$ (see also the metric (??) in this paper).

After fixing a natural realization $H^{*}$ we identify the space-time $M$ with a submanifold of $H^{*}$ consisting of delta-functions $\delta(x-a)$. This reduction is associated with the process of decoherence and collapse naturally happening under a continuous observation of the particle's position.

The submanifold $M$ is locally parameterized by the elements $a \in R^{4}$ which serve as abstract parameters needed to define the elements of $H^{*}$. The manifold structure on $M$ is not defined by the space of parameters, but appears as a restriction (pull-back) of the manifold structure on $H^{*}$. It is therefore defined by a specific realization $H^{*}$ of the abstract Hilbert space $\mathbf{S}$.

After the space $H^{*}$ and the submanifold $M$ are chosen, the parameters $a^{\mu}$ can be identified with coordinates on space-time, the vectors tangent to $M$ become identified with the ordinary space-time vectors and the induced metric becomes an (arbitrary) Riemannian metric on space-time.

As a clarifying example consider the Hilbert space generated by the delta-functions $\delta(\theta-a)$ on $R$ with functions $\delta(\theta-a)$ and $\delta(\theta-(a+2 \pi))$ identified for any $a \in R$. The space of parameters here is $R$. The space $H^{*}$ is a space of generalized functions on the circle $S^{1}$. The space $M$ consists of all delta-functions on $S^{1}$ and by the formalism of the last section is naturally identified with the unit circle itself. The parameter $a$ becomes then identified with the angular coordinate on $S^{1}$.

It is important to notice that the metric on space-time is derived from the metric on the Hilbert space $H^{*}$ which is, in turn, associated with a particular measurement performed on a macroscopic particle in the universe (see [5] for details). The variety of admissible metrics in the universe requires then a variety of realizations of the Hilbert space $\mathbf{S}$. This fact is in complete agreement with the coordinate formalism of [5].

In this interpretation a specific Riemannian structure, and, in fact, topology of the emerging space-time is determined by a specific large-scale measuring experiment performed in/on the universe. If one simply observes the results of measurements of positions of macroscopic particles naturally occurring in the universe, one ends up with the classical space-time as we know it. Assuming that a different experiment in/on the universe can be set, a different space-time shall, in general, come out of it.

Consider in particular a Hilbert space $H^{*}$ generated by all delta-functions on the product $R \times S^{3}$ of the real line and a 3 -sphere. More precisely, the elements of $H^{*}$ are functionals acting on a space of continuous functions on the cylinder $R \times S^{3}$. In this case the space-time $M$ has itself a topology of the cylinder. It is in principle conceivable that an experiment can be set that would result in such a topology of space-time. Even if such "global" experiments are not within our reach, a "local" change of topology of space is still a possibility. 
Notice once again that different topologies on the "emerging" space-time are produced simply by changing a particular functional realization of $\mathbf{S}$ and despite the fact that all such realizations are isomorphic. That is, what looks like a "coordinate transformation" on $\mathbf{S}$ (see [5]), can be observed as a change in topology on $M$.

The paper leaves many questions unanswered and, in fact, presents only the first look at the advocated approach to the problem of emergence. The results seem to be promising but clearly require further exploration. As no dynamics has been discussed, it is hard to compare the proposed "emergence formalism" to the existing approaches to emergence of the classical space-time (see [4] and references therein). In particular, it remains to be seen if the formalism is adequate for the dynamical treatment of the problem of emergence.

\section{References}

[1] Lang S 1985 Differentiable Manifolds (New York, Berlin, Heidelberg, Tokyo: Springer-Verlag)

[2] Kobayashi S and Nomizu K 1969 Foundations of Differential Geometry vol 2 (New York, London and Sydney: Interscience Publishers)

[3] Bohm A 1978 The Rigged Hilbert Space and Quantum Mechanics (New York: Springer)

[4] Giulini D et al 1996 Decoherence and the Appearence of a Classical World in Quantum Theory (Berlin: Springer)

[5] Kryukov A 2003 Found. Phys. 33 No 3

[6] Kryukov A 1997 Nonlin. Anal.Theor. Meth. and Appl. 30 pp 819-24 